J. Appl. Numer. Optim. 2 (2020), No. 2, pp. 171-186

Available online at http://jano.biemdas.com

https://doi.org/10.23952/jano.2.2020.2.04

\title{
STRONG CONVERGENCE OF TWO INERTIAL PROJECTION ALGORITHMS IN HILBERT SPACES
}

\author{
BING TAN ${ }^{1, *}$, SHANSHAN XU ${ }^{2}$ \\ ${ }^{1}$ Institute of Fundamental and Frontier Sciences, \\ University of Electronic Science and Technology of China, Chengdu, China \\ ${ }^{2}$ School of Mathematical Sciences, University of Electronic Science and Technology of China, Chengdu, China
}

\begin{abstract}
In this paper, we propose two inertial projection algorithms for finding a common solution of monotone variational inclusions and hierarchical fixed point problems of nonexpansive mappings. We obtain two strong convergence theorems under some suitable conditions in Hilbert spaces. In addition, we also give numerical examples to compare our algorithms with the existing ones. Numerical results show that our proposed algorithms are efficient and robust.
\end{abstract}

Keywords. Hierarchical fixed point problem; Inertial algorithm; Variational inclusion problem; Monotone operator; Strong convergence.

\section{INTRODUCTION}

Let $C$ be a nonempty convex closed set in a real Hilbert space $H$. The inner product and the inducted norm are represented by $\langle\cdot, \cdot\rangle$ and $\|\cdot\|$, respectively. Let $I$ be the identity mapping on $C$, and $P_{C}$ the metric projection of $H$ onto set $C$. For any $x \in H$, there exists a unique nearest point in $C$, denoted by $P_{C} x$, such that $\left\|x-P_{C} x\right\| \leq\|x-y\|, \forall y \in C . P_{C}$ is called the metric projection of $H$ onto $C$. One has $\left\|y-P_{C} x\right\|^{2}+\left\|x-P_{C} x\right\|^{2} \leq\|x-y\|^{2}, \forall x \in H, y \in C$. To begin with, let us recall the following concepts in convex and nonlinear analysis. For all $x, y \in C$, a mapping $A: C \rightarrow H$ is said to be (i) monotone if $\langle A x-A y, x-y\rangle \geq 0$; (ii) $k$-strongly monotone if there exists a positive constant $k$ such that $\langle A x-A y, x-y\rangle \geq k\|x-y\|^{2}$; (iii) $\chi$-inverse strongly monotone if there exists a positive constant $\chi$ such that $\langle A x-A y, x-y\rangle \geq \chi\|A x-A y\|^{2}$; (iv) $L$-Lipschitzian if there exists a positive constant $L$ such that $\|A x-A y\| \leq L\|x-y\|$. Let us also recall that a mapping $T: C \rightarrow C$ is said to (i) nonexpansive if $\|T x-T y\| \leq\|x-y\|$; (ii) firmly nonexpansive if $\|T x-T y\|^{2} \leq\langle T x-T y, x-y\rangle$. Let $G: H \rightarrow 2^{H}$ be a set-valued operator on $H$. $G$ is said to be monotone if and only if $\langle p-q, x-y\rangle \geq 0$ for any $x, y \in H, p \in G x$ and $q \in G y$. Recall that a mutivalued operator is said to be maximal if and only if its graph is not contained in the graph of any other monotone operator properly. Let $A: H \rightarrow H$ be a single-valued and $B: H \rightarrow 2^{H}$ be a multi-valued mappings. We consider the following monotone variational inclusion problem (in short, MVIP):

$$
\text { find } x^{*} \in H \quad \text { such that } 0 \in(A+B) x^{*} \text {. }
$$

\footnotetext{
${ }^{*}$ Corresponding author.
}

E-mail addresses: bingtan72@gmail.com (B. Tan), xss0702@gmail.com (S. Xu).

Received December 8, 2019; Accepted May 13, 2020.

(C)2020 Journal of Applied and Numerical Optimization 
Denote by $\Phi$ the solution set of MVIP (1.1). MVIP (1.1) includes some important applications arising in different areas, such as machine learning, image processing, transportation, and linear inverse problems, as special cases, see, for instance $[1,6,18,20,21]$ and the references therein. It is known that, for each $x \in H$, there exists a unique $u \in H$ such that $u \in(I+\mu B)^{-1} x$, where $\mu>0$. The operator $J_{\mu}^{B}:=(I+\mu B)^{-1}$ defined on $H$ is referred to as the resolvent of $B$, which is a single-valued and firmly nonexpansive mapping.

On the other hand, hierarchical fixed point problem (in short, HFPP) is an important problem which covers monotone variational inequality on fixed point sets, hierarchical minimization problems, minimization problems over equilibrium constraints, etc, see, e.g., [10, 13, 14]. In 2006, Moudafi and Mainge [15] introduced the following HFPP for a nonexpansive mapping $T$ with respect to another nonexpansive mapping $S$ on $C$, namely,

$$
\text { find } x^{*} \in \operatorname{Fix}(T) \quad \text { such that }\left\langle(I-S) x^{*}, x^{*}-x\right\rangle \leq 0, \quad \forall x \in \operatorname{Fix}(T),
$$

where $\operatorname{Fix}(T)$ is the set of fixed points of $T$, i.e., $\operatorname{Fix}(T):=\{x \in C: T x=x\}$. The solution set of HFPP (1.2) is represented as $\Psi:=\left\{x^{*} \in C: x^{*}=\left(P_{\mathrm{Fix}(T)} \circ S\right) x^{*}\right\}$. It is easy to check that solving HFPP (1.2) is equivalent to solving the fixed-point problem:

$$
\text { find } x^{*} \in C \quad \text { such that } x^{*}=P_{\mathrm{Fix}(T)} \circ S x^{*} \text {. }
$$

Let $N_{\mathrm{Fix}(T)}$ be the normal cone to $\operatorname{Fix}(T)$, which is defined by

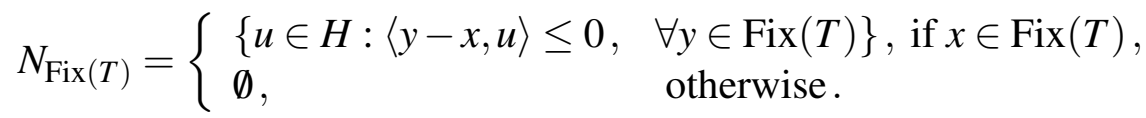

Then, HFPP (1.2) is equivalent to the variational inclusion problem:

$$
\text { find } x^{*} \in C \quad \text { such that } 0 \in N_{\operatorname{Fix}(T)} x^{*}+(I-S) x^{*} .
$$

Some authors have studied the hierarchical fixed point problem of nonlinear mappings and other related approximation problems, see, e.g., [5, 13, 14, 15, 19]. In 2007, Moudafi [14] introduced the following Mann iteration algorithm for solving HFPP (1.2):

$$
x_{n+1}=\left(1-\psi_{n}\right) x_{n}+\psi_{n}\left(v_{n} S x_{n}+\left(1-v_{n}\right) T x_{n}\right), \quad \forall n \geq 0,
$$

where $\left\{\psi_{n}\right\}$ and $\left\{v_{n}\right\}$ are two sequences in $(0,1)$. They proved the weak convergence of iterative algorithm (1.6). Iterative algorithm (1.6) can derive a number of algorithms, including the proximal methods and the gradient methods.

In 2015, Malitsky and Semenov [17] introduced a new hybrid method without extrapolation step for solving variational inequality problems. They obtained a strong convergence theorem and their numerical experiments show that their method has a competitive performance. Recently, Kazmi et al. [10] proved a strong convergence theorem with the following hybrid iterative method without extrapolating step for MVIP (1.1) and HFPP (1.2):

$$
\left\{\begin{array}{l}
y_{n}=\left(1-\psi_{n}\right) x_{n}+\psi_{n}\left(v_{n} S z_{n}+\left(1-v_{n}\right) T z_{n}\right), \\
z_{n+1}=J_{\mu}^{B}\left(y_{n}-\mu A y_{n}\right) \\
C_{n}=\left\{z \in C:\left\|z_{n+1}-z\right\|^{2} \leq\left(1-\psi_{n}\right)\left\|x_{n}-z\right\|^{2}+\psi_{n}\left\|z_{n}-z\right\|^{2}\right\} \\
Q_{n}=\left\{z \in C:\left\langle x_{n}-z, x_{n}-x_{0}\right\rangle \leq 0\right\} \\
x_{n+1}=P_{C_{n} \cap Q_{n}} x_{0}, n \geq 0 .
\end{array}\right.
$$


In recent years, inertial technology as an acceleration method has attracted extensive research. Many authors have established different fast iterative algorithms using inertial technology. These algorithms have shown advantages in theory and numerical experiments, and have been applied to fields such as image processing and machine learning. For more discussion, we recommend readers to refer to $[1,3,6,11,12,22]$ and the references therein.

In this paper, inspired and motivated by the above results, we introduce two inertial shrinking projection algorithms for solving MVIP (1.1) and HFPP (1.2). Strong convergence theorems are established in the framework of real Hilbert spaces. We also give some numerical examples to illustrate the computational performance of our proposed algorithms. Our methods improve and generalize some results presented by Mainge and Moudafi [13], Malitsky and Semenov [17], Kazmi et al. [10], Dong and Lu [8], Yuying and Plubtieng [24], Dong et al. [7], Tan, Xu and $\mathrm{Li}$ [23]. This paper is organized as follows. Section 2 gives the mathematical preliminaries. Section 3 and Section 4 are devoted to the two algorithms. Section 5, the last section, numerically compares the behaviors of the proposed algorithms and the existing ones.

\section{Preliminaries}

Let $\left\{x_{n}\right\}$ be a sequence in a Hilbert space $H$. Let $\omega_{w}\left\{x_{n}\right\}$ denote the set of all weak limits of $\left\{x_{n}\right\}$. One denotes the strong and weak convergence of $\left\{x_{n}\right\}$ to a point $x \in H$ by $x_{n} \rightarrow x$ and $x_{n} \rightarrow x$, respectively. For each $x, y, z \in H$, we have the following facts.

(1) $\|x+y\|^{2} \leq\|x\|^{2}+2\langle y, x+y\rangle$;

(2) $\|t x+(1-t) y\|^{2}=t\|x\|^{2}+(1-t)\|y\|^{2}-t(1-t)\|x-y\|^{2}, \quad \forall t \in R$;

(3) $\langle x-y, z\rangle=\frac{1}{2}\left[(x-y)^{2}+z^{2}-(x-y-z)^{2}\right]$.

The following lemmas play important roles in the proofs.

Lemma 2.1. [2] Let $T: C \rightarrow H$ be a nonexpansive mapping on $H$. Then

(i) $\operatorname{Fix}(T)$ is closed and convex;

(ii) $T$ is demiclosed on $H$, that is, if $x_{n} \rightarrow x$ and $T x_{n}-x_{n} \rightarrow 0$ as $n \rightarrow+\infty$, then $x \in \operatorname{Fix}(T)$.

Lemma 2.2. [8] Let $\left\{a_{n}\right\}$ and $\left\{\xi_{n}\right\}$ be nonnegative real sequences. Assume that the following inequality holds: $a_{n+1} \leq \alpha a_{n}+\beta \xi_{n}, \forall n \geq 1$, where $\alpha \in[0,1)$ and $\beta>0$. If $\sum_{n=1}^{\infty} \xi_{n}<+\infty$, then $\lim _{n \rightarrow \infty} a_{n}=0$.

Lemma 2.3. [16] Let $C$ be a nonempty convex closed set in a real Hilbert space $H$. Let $\left\{x_{n}\right\} \subset H, u \in H$ and $q=P_{C} u$. If the weak $\omega$-limit set $\omega_{w}\left\{x_{n}\right\} \subset C$ and satisfies the condition $\left\|x_{n}-u\right\| \leq\|u-q\|$, then $\left\{x_{n}\right\}$ converges strongly to $q$.

\section{THE INERTIAL SHRINKING PROJECTION AlgORITHM WITHOUT EXTRAPOLATING STEP}

Theorem 3.1. Let $C$ be a nonempty convex closed subset of a real Hilbert space $H$. Let $S, T: C \rightarrow C$ be two nonexpansive mappings. Let $A: C \rightarrow H$ be a $\chi$-inverse strongly monotone mapping and let $B: H \rightarrow 2^{H}$ be a set-valued maximal monotone operator. Assume that $\Upsilon=\Phi \bigcap \Psi \cap \operatorname{Fix}(\mathrm{S}) \neq \emptyset$ and the following conditions are satisfied:

(C1) $\left\{\delta_{n}\right\} \subset\left[\delta_{1}, \delta_{2}\right], \delta_{1} \in(-\infty, 0], \delta_{2} \in[0, \infty)$;

(C2) $\left\{\psi_{n}\right\} \subseteq(0, \psi], \psi \in\left(0, \frac{1}{1+\sigma}\right), \sigma \in(0,1), \lim _{n \rightarrow \infty} \inf \psi_{n}>0$;

(C3) $\left\{v_{n}\right\} \subseteq\left[v_{1}, v_{2}\right], v_{1}, v_{2} \in(0,1)$;

(C4) $\mu \in(0,2 \chi)$. 
Set $x_{-1}, x_{0}, z_{0} \in C$ arbitrarily. Define two sequences $\left\{x_{n}\right\}$ and $\left\{z_{n}\right\}$ by the following algorithm:

$$
\left\{\begin{array}{l}
w_{n}=x_{n}+\delta_{n}\left(x_{n}-x_{n-1}\right), \\
y_{n}=\left(1-\psi_{n}\right) w_{n}+\psi_{n}\left(v_{n} S z_{n}+\left(1-v_{n}\right) T z_{n}\right), \\
z_{n+1}=J_{\mu}^{B}\left(y_{n}-\mu A y_{n}\right), \\
C_{n+1}=\left\{p \in C_{n}:\left\|z_{n+1}-p\right\|^{2} \leq\left(1-\psi_{n}\right)\left\|w_{n}-p\right\|^{2}+\psi_{n}\left\|z_{n}-p\right\|^{2}\right\}, \\
x_{n+1}=P_{C_{n+1}} x_{0}, n \geq 0 .
\end{array}\right.
$$

Then the sequences $\left\{x_{n}\right\}$ and $\left\{z_{n}\right\}$ defined by (3.1) converge strongly to $x^{*} \in \Upsilon$, where $x^{*}=P_{\Upsilon} x_{0}$.

Proof. Our proof is divided into four steps.

Step 1. We show that $\Upsilon \subset C_{n+1}$ for all $n \geq 0$. Since $A: C \rightarrow H$ is a $\chi$-inverse strongly monotone mapping, we get that $(I-\mu A)$ is nonexpansive. Hence $J_{\mu}^{B}(I-\mu A)$ is also nonexpansive. Since $\Upsilon \neq \emptyset$, it follows from Lemma 2.1 (i) that $\Phi=$ Fix $\left(J_{\mu}^{B}(I-\mu A)\right)$ is convex and closed. Hence Fix $\left(J_{\mu}^{B}(I-\mu A)\right)=(A+B)^{-1}(0)$ is convex and closed. Since $\Psi=\operatorname{Fix}\left(P_{\operatorname{Fix}(T)} \circ S\right) \neq \emptyset$, we observe that $\Psi$ is convex and closed. Therefore, $\Upsilon$ is a nonempty convex and closed set. Hence $P_{\Upsilon} x_{0}$ is well defined. Further, we can easily observe that $C_{n+1}$ is convex and closed. Next, we claim that $\Upsilon \subset C_{n+1}, \forall n \geq 0$. Indeed, for any $u \in \Upsilon$, we have

$$
\begin{aligned}
\left\|y_{n}-u\right\|^{2}= & \left\|\left(1-\psi_{n}\right) w_{n}+\psi_{n}\left(v_{n} S z_{n}+\left(1-v_{n}\right) T z_{n}\right)-u\right\|^{2} \\
\leq & \left(1-\psi_{n}\right)\left\|w_{n}-u\right\|^{2}+\psi_{n}\left(v_{n}\left\|S z_{n}-u\right\|^{2}+\left(1-v_{n}\right)\left\|T z_{n}-u\right\|^{2}\right. \\
& \left.-v_{n}\left(1-v_{n}\right)\left\|S z_{n}-T z_{n}\right\|^{2}\right) \\
\leq & \left(1-\psi_{n}\right)\left\|w_{n}-u\right\|^{2}+\psi_{n}\left(v_{n}\left\|z_{n}-u\right\|^{2}+\left(1-v_{n}\right)\left\|z_{n}-u\right\|^{2}\right. \\
& \left.-v_{n}\left(1-v_{n}\right)\left\|S z_{n}-T z_{n}\right\|^{2}\right) \\
\leq & \left(1-\psi_{n}\right)\left\|w_{n}-u\right\|^{2}+\psi_{n}\left\|z_{n}-u\right\|^{2}-\psi_{n} v_{n}\left(1-v_{n}\right)\left\|S z_{n}-T z_{n}\right\|^{2} \\
\leq & \left(1-\psi_{n}\right)\left\|w_{n}-u\right\|^{2}+\psi_{n}\left\|z_{n}-u\right\|^{2} .
\end{aligned}
$$

Since $u \in \Upsilon$, then $u \in(A+B)^{-1}(0)$ and hence $J_{\mu}^{B}(I-\mu A) u=u$. Therefore,

$$
\begin{aligned}
\left\|z_{n+1}-u\right\|^{2} & =\left\|J_{\mu}^{B}\left(y_{n}-\mu A y_{n}\right)-J_{\mu}^{B}(u-\mu A u)\right\|^{2} \\
& \leq\left\|\left(y_{n}-u\right)-\mu\left(A y_{n}-A u\right)\right\|^{2} \\
& =\left\|y_{n}-u\right\|^{2}+\mu^{2}\left\|A y_{n}-A u\right\|^{2}-2 \mu\left\langle y_{n}-u, A y_{n}-A u\right\rangle \\
& \leq\left\|y_{n}-u\right\|^{2}+\mu^{2}\left\|A y_{n}-A u\right\|^{2}-2 \mu \chi\left\|A y_{n}-A u\right\|^{2} \\
& \leq\left\|y_{n}-u\right\|^{2} .
\end{aligned}
$$

Combining (3.2) and (3.3), we see that

$$
\left\|z_{n+1}-u\right\|^{2} \leq\left(1-\psi_{n}\right)\left\|w_{n}-u\right\|^{2}+\psi_{n}\left\|z_{n}-u\right\|^{2}
$$

which implies that $u \in C_{n+1}$ and hence $\Upsilon \subset C_{n+1}$ for all $n \geq 0$.

Step 2. We show that $\left\{x_{n}\right\}$ converges weakly to $x^{*} \in(A+B)^{-1}(0), x^{*} \in \operatorname{Fix}(T)$ and $x^{*} \in \operatorname{Fix}(S)$. Since $x_{n}=P_{C_{n}} x_{0}$, this together with $\Upsilon \subset C_{n}$ implies $\left\|x_{n}-x_{0}\right\| \leq\left\|u-x_{0}\right\|, \forall u \in \Upsilon$, which implies that $\left\{x_{n}\right\}$ is bounded. In particular,

$$
\left\|x_{n}-x_{0}\right\| \leq\left\|x^{*}-x_{0}\right\|, \quad \text { where } x^{*}=P_{\Upsilon} x_{0} .
$$


Since $x_{n}=P_{C_{n}} x_{0}$ and $x_{n+1} \in C_{n+1} \subset C_{n}$, we obtain

$$
\left\|x_{n}-x_{n+1}\right\|^{2} \leq\left\|x_{n+1}-x_{0}\right\|^{2}-\left\|x_{n}-x_{0}\right\|^{2}, \quad \forall n \geq 0 .
$$

(3.5) and (3.6) lead us to

$$
\begin{aligned}
\sum_{n=1}^{N}\left\|x_{n+1}-x_{n}\right\|^{2} & \leq \sum_{n=1}^{N}\left(\left\|x_{n+1}-x_{0}\right\|^{2}-\left\|x_{n}-x_{0}\right\|^{2}\right) \\
& =\left\|x_{N+1}-x_{0}\right\|^{2}-\left\|x_{1}-x_{0}\right\|^{2} \\
& \leq\left\|x^{*}-x_{0}\right\|^{2}-\left\|x_{1}-x_{0}\right\|^{2}
\end{aligned}
$$

which implies that $\sum_{n=1}^{\infty}\left\|x_{n+1}-x_{n}\right\|^{2}$ is convergent. Hence

$$
\lim _{n \rightarrow \infty}\left\|x_{n+1}-x_{n}\right\|=0 .
$$

Next, by the definition of $\left\{w_{n}\right\}$ in (3.1) and condition (C1), we have

$$
\left\|w_{n}-x_{n}\right\|=\delta_{n}\left\|x_{n}-x_{n-1}\right\| \leq \max \left\{\left|\delta_{1}\right|,\left|\delta_{2}\right|\right\}\left\|x_{n}-x_{n-1}\right\| \rightarrow 0 .
$$

It follows from (3.7) and (3.8) that

$$
\left\|w_{n}-x_{n+1}\right\| \leq\left\|w_{n}-x_{n}\right\|+\left\|x_{n}-x_{n+1}\right\| \rightarrow 0 .
$$

On the other hand, by the definition of $\left\{w_{n}\right\}$ in (3.1), we also have

$$
\begin{aligned}
\left\|w_{n}-x_{n+1}\right\|^{2} & =\left\|x_{n}-x_{n+1}+\delta_{n}\left(x_{n}-x_{n-1}\right)\right\|^{2} \\
& =\left\|x_{n}-x_{n+1}\right\|^{2}+\delta_{n}^{2}\left\|x_{n}-x_{n-1}\right\|^{2}+2 \delta_{n}\left\langle x_{n}-x_{n+1}, x_{n}-x_{n-1}\right\rangle \\
& \leq\left\|x_{n}-x_{n+1}\right\|^{2}+\delta_{n}^{2}\left\|x_{n}-x_{n-1}\right\|+\delta_{n}\left[\left\|x_{n}-x_{n+1}\right\|^{2}+\left\|x_{n}-x_{n-1}\right\|^{2}\right] \\
& \leq\left(1+\delta_{n}\right)\left\|x_{n}-x_{n+1}\right\|^{2}+\delta_{n}\left(1+\delta_{n}\right)\left\|x_{n}-x_{n-1}\right\|^{2} .
\end{aligned}
$$

From (3.10), using $x_{n+1} \in C_{n+1}$ and condition (C2), we obtain

$$
\begin{aligned}
\left\|z_{n+1}-x_{n+1}\right\|^{2} \leq & \left(1-\psi_{n}\right)\left\|w_{n}-x_{n+1}\right\|^{2}+\psi_{n}\left\|z_{n}-x_{n+1}\right\|^{2} \\
\leq & \left(1-\psi_{n}\right)\left[\left(1+\delta_{n}\right)\left\|x_{n}-x_{n+1}\right\|^{2}+\delta_{n}\left(1+\delta_{n}\right)\left\|x_{n}-x_{n-1}\right\|^{2}\right] \\
& +\psi_{n}\left[\left\|z_{n}-x_{n}\right\|^{2}+\left\|x_{n}-x_{n+1}\right\|^{2}+2\left\langle z_{n}-x_{n}, x_{n}-x_{n+1}\right\rangle\right] \\
\leq & \left(1-\psi_{n}\right)\left[\left(1+\delta_{n}\right)\left\|x_{n}-x_{n+1}\right\|^{2}+\delta_{n}\left(1+\delta_{n}\right)\left\|x_{n}-x_{n-1}\right\|^{2}\right] \\
& +\psi_{n}\left[\left(1+\sigma^{2}\right)\left\|z_{n}-x_{n}\right\|^{2}+\left(1+\frac{1}{\sigma^{2}}\right)\left\|x_{n}-x_{n+1}\right\|^{2}\right] \\
\leq & \varphi^{*}\left\|z_{n}-x_{n}\right\|^{2}+\left(\delta_{2}+\frac{1+2 \sigma^{2}}{\sigma^{2}}\right)\left\|x_{n}-x_{n+1}\right\|^{2}+\delta_{2}\left(1+\delta_{2}\right)\left\|x_{n}-x_{n-1}\right\|^{2} \\
\leq & \varphi^{*}\left\|z_{n}-x_{n}\right\|^{2}+\xi_{n},
\end{aligned}
$$

where $\varphi^{*}=\psi_{n}(1+\sigma)<1$ and

$$
\xi_{n}=\left(\delta_{2}+\frac{1+2 \sigma^{2}}{\sigma^{2}}\right)\left\|x_{n}-x_{n+1}\right\|^{2}+\delta_{2}\left(1+\delta_{2}\right)\left\|x_{n}-x_{n-1}\right\|^{2} .
$$


From $\sum_{n=1}^{\infty}\left\|x_{n+1}-x_{n}\right\|^{2}<+\infty$, one has $\sum_{n=1}^{\infty} \xi_{n}<\infty$. Using Lemma 2.2 in (3.11) yields that

$$
\lim _{n \rightarrow \infty}\left\|z_{n}-x_{n}\right\|=0 \text {. }
$$

Since $\left\{x_{n}\right\}$ is bounded, one finds that (3.12), (3.9), and (3.2) imply that $\left\{z_{n}\right\},\left\{w_{n}\right\}$ and $\left\{y_{n}\right\}$ are also bounded, respectively. From (3.7) and (3.12), one obtains that

$$
\left\|z_{n+1}-x_{n}\right\| \leq\left\|z_{n+1}-x_{n+1}\right\|+\left\|x_{n+1}-x_{n}\right\| \rightarrow 0 .
$$

Combining (3.8) and (3.12), one has

$$
\left\|z_{n}-w_{n}\right\| \leq\left\|z_{n}-x_{n}\right\|+\left\|x_{n}-w_{n}\right\| \rightarrow 0 .
$$

It follows from (3.8) and (3.13), one obtains that

$$
\left\|z_{n+1}-w_{n}\right\| \leq\left\|z_{n+1}-x_{n}\right\|+\left\|x_{n}-w_{n}\right\| \rightarrow 0 .
$$

Next, from (3.2) and (3.3), one sees that

$$
\begin{aligned}
\left\|z_{n+1}-u\right\|^{2} & \leq\left\|y_{n}-u\right\|^{2}-\mu(2 \chi-\mu)\left\|A y_{n}-A u\right\|^{2} \\
& \leq\left(1-\psi_{n}\right)\left\|w_{n}-u\right\|^{2}+\psi_{n}\left\|z_{n}-u\right\|^{2}-\mu(2 \chi-\mu)\left\|A y_{n}-A u\right\|^{2},
\end{aligned}
$$

which implies

$$
\begin{aligned}
\mu(2 \chi-\mu)\left\|A y_{n}-A u\right\|^{2} \leq & \left\|w_{n}-u\right\|^{2}-\left\|z_{n+1}-u\right\|^{2}+\psi_{n}\left(\left\|z_{n}-u\right\|^{2}-\left\|w_{n}-u\right\|^{2}\right) \\
\leq & \left\|w_{n}-z_{n+1}\right\|\left(\left\|w_{n}-u\right\|+\left\|z_{n+1}-u\right\|\right) \\
& +\psi_{n}\left\|z_{n}-w_{n}\right\|\left(\left\|z_{n}-u\right\|+\left\|w_{n}-u\right\|\right) \\
\leq & L_{1}\left\|w_{n}-z_{n+1}\right\|+\psi_{n} L_{2}\left\|z_{n}-w_{n}\right\|,
\end{aligned}
$$

where $L_{1}:=\sup _{n \geq 0}\left\{\left\|w_{n}-u\right\|+\left\|z_{n+1}-u\right\|\right\}, L_{2}:=\sup _{n \geq 0}\left\{\left\|z_{n}-u\right\|+\left\|w_{n}-u\right\|\right\}$. In view of $\mu(2 \chi-\mu)>0,(3.14),(3.15),(3.16)$ and condition (C2), one arrives at

$$
\lim _{n \rightarrow \infty}\left\|A y_{n}-A u\right\|=0 \text {. }
$$

Since $J_{\mu}^{B}$ is firmly nonexpansive, one has

$$
\begin{aligned}
\left\|z_{n+1}-u\right\|^{2} & =\left\|J_{\mu}^{B}(I-\mu A) y_{n}-J_{\mu}^{B}(I-\mu A) u\right\|^{2} \\
& \leq\left\langle z_{n+1}-u,(I-\mu A) y_{n}-(I-\mu A) u\right\rangle \\
& =\frac{1}{2}\left[\left\|(I-\mu A) y_{n}-(I-\mu A) u\right\|^{2}+\left\|z_{n+1}-u\right\|^{2}-\left\|y_{n}-z_{n+1}-\mu\left(A y_{n}-A u\right)\right\|^{2}\right] \\
& \leq \frac{1}{2}\left[\left\|y_{n}-u\right\|^{2}+\left\|z_{n+1}-u\right\|^{2}-\left\|y_{n}-z_{n+1}\right\|^{2}+2 \mu\left\|y_{n}-z_{n+1}\right\|\left\|A y_{n}-A u\right\|\right]
\end{aligned}
$$

which in turn yields

$$
\left\|z_{n+1}-u\right\|^{2} \leq\left\|y_{n}-u\right\|^{2}-\left\|y_{n}-z_{n+1}\right\|^{2}+2 \mu\left\|y_{n}-z_{n+1}\right\|\left\|A y_{n}-A u\right\| .
$$

Combining (3.2), (3.16) and (3.19), we can show that

$$
\begin{aligned}
\left\|y_{n}-z_{n+1}\right\|^{2} \leq & \left(1-\psi_{n}\right)\left\|w_{n}-u\right\|^{2}+\psi_{n}\left\|z_{n}-u\right\|^{2}-\left\|z_{n+1}-u\right\|^{2} \\
& +2 \mu\left\|y_{n}-z_{n+1}\right\|\left\|A y_{n}-A u\right\| \\
\leq & L_{1}\left\|w_{n}-z_{n+1}\right\|+\psi_{n} L_{2}\left\|z_{n}-w_{n}\right\|+2 \mu L_{3}\|\| A y_{n}-A u \|,
\end{aligned}
$$


where $L_{3}:=\sup _{n}\left\{\left\|y_{n}-z_{n+1}\right\|\right\}$. Using (3.14), (3.15), (3.17), (3.20) and condition (C2), one has

$$
\lim _{n \rightarrow \infty}\left\|y_{n}-z_{n+1}\right\|=0 \text {. }
$$

It follows from (3.15) and (3.21) that

$$
\left\|y_{n}-w_{n}\right\| \leq\left\|y_{n}-z_{n+1}\right\|+\left\|z_{n+1}-w_{n}\right\| \rightarrow 0 .
$$

Combining (3.14) and (3.22), we see that

$$
\lim _{n \rightarrow \infty}\left\|w_{n}-y_{n}-\psi_{n}\left(w_{n}-z_{n}\right)\right\|=0 .
$$

From (3.2) and (3.3), we have

$$
\begin{aligned}
\psi_{n} v_{n}\left(1-v_{n}\right)\left\|S z_{n}-T z_{n}\right\|^{2} & \leq\left\|w_{n}-u\right\|^{2}-\left\|z_{n+1}-u\right\|^{2}+\psi_{n}\left(\left\|z_{n}-u\right\|^{2}-\left\|w_{n}-u\right\|^{2}\right) \\
& \leq L_{1}\left\|w_{n}-z_{n+1}\right\|+\psi_{n} L_{2}\left\|z_{n}-w_{n}\right\| .
\end{aligned}
$$

Using (3.14), (3.15), conditions (C2) and (C3), we obtain

$$
\lim _{n \rightarrow \infty}\left\|S z_{n}-T z_{n}\right\|=0 .
$$

Further, from (3.1), it follows that

$$
\left\|T z_{n}-z_{n}\right\| \leq \frac{1}{\psi_{n}}\left\|y_{n}-w_{n}\right\|+\left\|w_{n}-z_{n}\right\|+v_{n}\left\|T z_{n}-S z_{n}\right\| .
$$

Combining (3.14), (3.22), (3.24), (3.25), conditions (C2) and (C3), we obtain

$$
\lim _{n \rightarrow \infty}\left\|z_{n}-T z_{n}\right\|=0 \text {. }
$$

By means of (3.24) and (3.26), we have

$$
\lim _{n \rightarrow \infty}\left\|S z_{n}-z_{n}\right\|=0 .
$$

Since $\left\{x_{n}\right\}$ is bounded, there exists a subsequence $\left\{x_{n_{i}}\right\}$ of $\left\{x_{n}\right\}$ such that $x_{n_{i}} \rightarrow x^{*}$. Further, it follows from (3.8), (3.12), (3.22) that the sequences $\left\{x_{n}\right\},\left\{w_{n}\right\},\left\{y_{n}\right\}$ and $\left\{z_{n}\right\}$ all have the same asymptotic behavior. Therefore, combining (3.21), (3.26) and (3.27), we obtain from Lemma 2.1 (ii) that $x^{*} \in(A+B)^{-1}(0), x^{*} \in \operatorname{Fix}(T)$ and $x^{*} \in \operatorname{Fix}(S)$.

Step 3. We show that $x^{*} \in \Upsilon$. From (3.1), we have

$$
y_{n}-w_{n}=\psi_{n}\left(z_{n}-w_{n}\right)+\psi_{n}\left(v_{n}\left(S z_{n}-z_{n}\right)+\left(1-v_{n}\right)\left(T z_{n}-z_{n}\right)\right),
$$

therefore,

$$
\frac{1}{\psi_{n} v_{n}}\left(w_{n}-y_{n}-\psi_{n}\left(w_{n}-z_{n}\right)\right)=(I-S) z_{n}+\left(\frac{1-v_{n}}{v_{n}}\right)(I-T) z_{n} .
$$

Thus, using monotonicity of $I-S$, it follows that

$$
\begin{aligned}
& \left\langle\frac{1}{\psi_{n} v_{n}}\left(w_{n}-y_{n}-\psi_{n}\left(w_{n}-z_{n}\right)\right), z_{n}-p\right\rangle \\
= & \left\langle(I-S) z_{n}-(I-S) p, z_{n}-p\right\rangle+\left\langle(I-S) p, z_{n}-p\right\rangle+\frac{1-v_{n}}{v_{n}}\left\langle z_{n}-T z_{n}, z_{n}-p\right\rangle \\
\geq & \left\langle(I-S) p, z_{n}-p\right\rangle+\frac{1-v_{n}}{v_{n}}\left\langle z_{n}-T z_{n}, z_{n}-p\right\rangle, \quad \forall p \in \operatorname{Fix}(T) .
\end{aligned}
$$

Using (3.23), (3.26), conditions (C2) and (C3) in (3.29), we obtain

$$
\limsup _{n \rightarrow \infty}\left\langle p-S p, z_{n}-p\right\rangle \leq 0, \quad \forall p \in \operatorname{Fix}(T) .
$$


Since $z_{n} \rightarrow x^{*}$, it follows from (3.30) that

$$
\left\langle(I-S) p, x^{*}-p\right\rangle \leq 0, \quad \forall p \in \operatorname{Fix}(T)
$$

According to $\operatorname{Fix}(T)$ is convex, one has $\tau p+(1-\tau) x^{*} \in \operatorname{Fix}(T)$ for $\tau \in(0,1)$. Thus

$$
\begin{aligned}
& \left\langle(I-S)\left(\tau p+(1-\tau) x^{*}\right), x^{*}-\left(\tau p+(1-\tau) x^{*}\right)\right\rangle \\
& =\tau\left\langle(I-S)\left(\tau p+(1-\tau) x^{*}\right), x^{*}-p\right\rangle \\
& \leq 0, \quad \forall p \in \operatorname{Fix}(T),
\end{aligned}
$$

which implies $\left\langle(I-S)\left(\tau p+(1-\tau) x^{*}\right), x^{*}-p\right\rangle \leq 0, \forall p \in \operatorname{Fix}(T)$. On taking limits $\tau \rightarrow 0_{+}$, we have $\left\langle(I-S) x^{*}, x^{*}-p\right\rangle \leq 0, \forall p \in \operatorname{Fix}(T)$. That is $x^{*} \in \Psi$ and thus $x^{*} \in \Upsilon$.

Step 4. Finally, we show that $x_{n} \rightarrow x^{*}$, where $x^{*}=P_{\Upsilon} x_{0}$. Since $\omega_{w}\left\{x_{n}\right\} \subset \Upsilon$, we see that (3.5) and Lemma 2.3 ensure the strong convergence of $\left\{x_{n}\right\}$ to $x^{*}$, where $x^{*}=P_{\Upsilon} x_{0}$.

Remark 3.1. (i) If we modify the definitions of $\left\{y_{n}\right\}$ and $C_{n+1}$ in the iterative Algorithm (3.1), just like the reference [10] did, we can get a new algorithm different from Algorithm (3.1).

(ii) If we set $B=\partial I_{C}$ and $A=0$, then we obtain a new inertial shrinking projection algorithm without extrapolating step for solving HFPP (1.2), which was proposed by Tan et al. [23].

\section{THE INERTIAL SHRINKING PROJECTION AlgORITHM}

Theorem 4.1. Let $C$ be a nonempty convex and closed set in a real Hilbert space $H$. Let $S, T: C \rightarrow C$ be two nonexpansive mappings. Let $A: C \rightarrow H$ be a $\chi$-inverse strongly monotone mapping and let $B: H \rightarrow 2^{H}$ be a set-valued maximal monotone operator. Assume that $\Upsilon=\Phi \bigcap \Psi \cap \operatorname{Fix}(\mathrm{S}) \neq \emptyset$ and the following conditions are satisfied:

(D1) $\left\{\delta_{n}\right\} \subset\left[\delta_{1}, \delta_{2}\right], \delta_{1} \in(-\infty, 0], \delta_{2} \in[0, \infty)$;

(D2) $\left\{\psi_{n}\right\} \subseteq(0, \psi], \psi \in\left(0, \frac{1}{1+\sigma}\right), \sigma \in(0,1), \lim _{n \rightarrow \infty} \inf \psi_{n}>0$;

(D3) $\left\{v_{n}\right\} \subseteq\left[v_{1}, v_{2}\right], v_{1}, v_{2} \in(0,1)$;

(D4) $\mu \in(0,2 \chi)$.

Set $x_{-1}, x_{0} \in C$ arbitrarily. Define two sequences $\left\{x_{n}\right\}$ and $\left\{z_{n}\right\}$ by the following algorithm:

$$
\left\{\begin{array}{l}
w_{n}=x_{n}+\delta_{n}\left(x_{n}-x_{n-1}\right), \\
y_{n}=\left(1-\psi_{n}\right) w_{n}+\psi_{n}\left(v_{n} S w_{n}+\left(1-v_{n}\right) T w_{n}\right), \\
z_{n}=J_{\mu}^{B}\left(y_{n}-\mu A y_{n}\right), \\
C_{n+1}=\left\{z \in C_{n}:\left\|z_{n}-z\right\|^{2} \leq\left\|w_{n}-z\right\|^{2}\right\} \\
x_{n+1}=P_{C_{n+1}} x_{0}, n \geq 0 .
\end{array}\right.
$$

Then the sequences $\left\{x_{n}\right\}$ and $\left\{z_{n}\right\}$ defined by (4.1) converge strongly to $x^{*} \in \Upsilon$, where $x^{*}=P_{\Upsilon} x_{0}$.

Proof. Our proof is divided into four steps.

Step 1. According to the Step 1 in Theorem 3.1, we see that $\Upsilon$ is a nonempty convex and closed set. Hence $P_{\Upsilon} x_{0}$ is well defined. Observe that $C_{n+1}$ is convex and closed. Next, we claim 
that $\Upsilon \subset C_{n+1}, \forall n \geq 0$. Indeed, for any $u \in \Upsilon$, we have

$$
\begin{aligned}
\left\|y_{n}-u\right\|^{2}= & \left\|\left(1-\psi_{n}\right) w_{n}+\psi_{n}\left(v_{n} S w_{n}+\left(1-v_{n}\right) T w_{n}\right)-u\right\|^{2} \\
= & \left\|\left(1-\psi_{n}\right)\left(w_{n}-u\right)+\psi_{n}\left(v_{n}\left(S w_{n}-u\right)+\left(1-v_{n}\right)\left(T w_{n}-u\right)\right)\right\|^{2} \\
\leq & \left(1-\psi_{n}\right)\left\|w_{n}-u\right\|^{2}+\psi_{n}\left(v_{n}\left\|S w_{n}-u\right\|^{2}\right. \\
& \left.+\left(1-v_{n}\right)\left\|T w_{n}-u\right\|^{2}-v_{n}\left(1-v_{n}\right)\left\|S w_{n}-T w_{n}\right\|^{2}\right) \\
\leq & \left\|w_{n}-u\right\|^{2}-\psi_{n} v_{n}\left(1-v_{n}\right)\left\|S w_{n}-T w_{n}\right\|^{2} .
\end{aligned}
$$

On the other hand, one has

$$
\left\|z_{n}-u\right\|^{2} \leq\left\|y_{n}-u\right\|^{2}-\mu(2 \chi-\mu)\left\|A y_{n}-A u\right\|^{2} \leq\left\|y_{n}-u\right\|^{2} .
$$

Combining (4.2) and (4.3), one has $\left\|z_{n}-u\right\|^{2} \leq\left\|w_{n}-u\right\|^{2}$. This implies that $u \in C_{n+1}$ and hence $\Upsilon \subset C_{n+1}$ for all $n \geq 0$.

Step 2. We show that $\left\{x_{n}\right\}$ converges weakly to $x^{*} \in(A+B)^{-1}(0), x^{*} \in \operatorname{Fix}(T)$ and $x^{*} \in \operatorname{Fix}(S)$. Using the same arguments as the Step 2 in Theorem 3.1, we can obtain that the sequence $\left\{x_{n}\right\}$ is bounded. Further, we can also prove that

$$
\sum_{n=1}^{\infty}\left\|x_{n+1}-x_{n}\right\|^{2}<+\infty, \quad \text { and } \quad \lim _{n \rightarrow \infty}\left\|x_{n+1}-x_{n}\right\|=0
$$

and

$$
\lim _{n \rightarrow \infty}\left\|w_{n}-x_{n}\right\|=0, \quad \text { and } \quad \lim _{n \rightarrow \infty}\left\|w_{n}-x_{n+1}\right\|=0 .
$$

In view of the $x_{n+1} \in C_{n+1}$, we obtain

$$
\left\|z_{n}-x_{n+1}\right\|^{2} \leq\left\|w_{n}-x_{n+1}\right\|^{2} .
$$

It follows from (4.5) and (4.6) that

$$
\lim _{n \rightarrow \infty}\left\|z_{n}-x_{n+1}\right\|=0 .
$$

Furthermore, combining (4.4) and (4.7), we see that

$$
\left\|z_{n}-x_{n}\right\| \leq\left\|z_{n}-x_{n+1}\right\|+\left\|x_{n+1}-x_{n}\right\| \rightarrow 0 .
$$

Using (4.5) and (4.8), one has

$$
\left\|z_{n}-w_{n}\right\| \leq\left\|z_{n}-x_{n}\right\|+\left\|x_{n}-w_{n}\right\| \rightarrow 0 .
$$

From (4.2), (4.3), (4.9), conditions (D2) and (D3), we have

$$
\begin{aligned}
\psi_{n} v_{n}\left(1-v_{n}\right)\left\|S w_{n}-T w_{n}\right\|^{2} & \leq\left\|w_{n}-u\right\|^{2}-\left\|y_{n}-u\right\|^{2} \leq\left\|w_{n}-u\right\|^{2}-\left\|z_{n}-u\right\|^{2} \\
& \leq\left\|w_{n}-z_{n}\right\|\left(\left\|w_{n}-u\right\|+\left\|z_{n}-u\right\|\right)=M\left\|w_{n}-z_{n}\right\|
\end{aligned}
$$

where $M:=\sup _{n \geq 0}\left\{\left\|w_{n}-u\right\|+\left\|z_{n}-u\right\|\right\}$. From (4.9), one has

$$
\lim _{n \rightarrow \infty}\left\|S w_{n}-T w_{n}\right\|=0 \text {. }
$$

According to (4.3), we have

$$
\mu(2 \chi-\mu)\left\|A y_{n}-A u\right\|^{2} \leq\left\|w_{n}-u\right\|^{2}-\left\|z_{n}-u\right\|^{2} \leq M\left\|w_{n}-z_{n}\right\| .
$$

Since $\mu(2 \chi-\mu)>0$, one concludes from (4.9) that

$$
\lim _{n \rightarrow \infty}\left\|A y_{n}-A u\right\|=0 .
$$


As the same in (3.18), we can show that

$$
\left\|z_{n}-u\right\|^{2} \leq\left\|y_{n}-u\right\|^{2}-\left\|y_{n}-z_{n}\right\|^{2}+2 \mu\left\|y_{n}-z_{n}\right\|\left\|A y_{n}-A u\right\|,
$$

which together with (4.2) gives that

$$
\begin{aligned}
\left\|y_{n}-z_{n}\right\|^{2} & \leq\left\|w_{n}-u\right\|^{2}-\left\|z_{n}-u\right\|^{2}+2 \mu\left\|y_{n}-z_{n}\right\|\left\|A y_{n}-A u\right\| \\
& \leq M\left\|w_{n}-z_{n}\right\|+2 \mu M_{1}\|\| A y_{n}-A u \|,
\end{aligned}
$$

where $M_{1}:=\sup _{n \geq 0}\left\{\left\|y_{n}-z_{n}\right\|\right\}$. Thus, it follows from (4.9) and (4.12) that

$$
\lim _{n \rightarrow \infty}\left\|y_{n}-z_{n}\right\|=0 \text {. }
$$

Combining (4.9) and (4.15), we obtain

$$
\left\|y_{n}-w_{n}\right\| \leq\left\|y_{n}-z_{n}\right\|+\left\|z_{n}-w_{n}\right\| \rightarrow 0 .
$$

Further, from (4.1), we have

$$
\left\|T w_{n}-w_{n}\right\| \leq \frac{1}{\psi_{n}}\left\|y_{n}-w_{n}\right\|+v_{n}\left\|T w_{n}-S w_{n}\right\|
$$

Hence, it follows from (4.10) and (4.16) that

$$
\lim _{n \rightarrow \infty}\left\|T w_{n}-w_{n}\right\|=0 .
$$

Combining (4.10) and (4.17), we see that

$$
\lim _{n \rightarrow \infty}\left\|S w_{n}-w_{n}\right\|=0 .
$$

Since

$$
\begin{aligned}
\left\|T x_{n}-x_{n}\right\| & \leq\left\|T x_{n}-T w_{n}\right\|+\left\|T w_{n}-w_{n}\right\|+\left\|w_{n}-x_{n}\right\| \\
& \leq 2\left\|w_{n}-x_{n}\right\|+\left\|T w_{n}-w_{n}\right\|,
\end{aligned}
$$

we obtain from (4.5) and (4.17) that

$$
\lim _{n \rightarrow \infty}\left\|T x_{n}-x_{n}\right\|=0 .
$$

Thus, it follows from (4.19) and Lemma 2.1 (ii) that every weak limit point of $\left\{x_{n}\right\}$ is a fixed point of mapping $T$, i.e., $\omega_{w}\left\{x_{n}\right\} \subset \operatorname{Fix}(T)$. Therefore, $\left\{x_{n}\right\}$ converges weakly to $x^{*} \in \operatorname{Fix}(T)$. Further, from (4.5), (4.15), (4.16), (4.17), (4.18), we obtain that $x^{*} \in(A+B)^{-1}(0), x^{*} \in \operatorname{Fix}(T)$ and $x^{*} \in \operatorname{Fix}(S)$.

Step 3. We show that $x^{*} \in \Upsilon$. From (4.1), we have

$$
y_{n}-w_{n}=\psi_{n}\left(v_{n}\left(S w_{n}-w_{n}\right)+\left(1-v_{n}\right)\left(T w_{n}-w_{n}\right)\right),
$$

and hence

$$
\frac{1}{\psi_{n} v_{n}}\left(w_{n}-y_{n}\right)=(I-S) w_{n}+\left(\frac{1-v_{n}}{v_{n}}\right)(I-T) w_{n} .
$$

Therefore, using monotonicity of $I-S$, we obtain

$$
\begin{aligned}
\left\langle\frac{w_{n}-y_{n}}{\psi_{n} v_{n}}, w_{n}-p\right\rangle= & \left\langle(I-S) w_{n}-(I-S) p, w_{n}-p\right\rangle+\left\langle(I-S) p, w_{n}-p\right\rangle \\
& +\frac{1-v_{n}}{v_{n}}\left\langle w_{n}-T w_{n}, w_{n}-p\right\rangle \\
\geq & \left\langle(I-S) p, w_{n}-p\right\rangle+\frac{1-v_{n}}{v_{n}}\left\langle w_{n}-T w_{n}, w_{n}-p\right\rangle, \quad \forall p \in \operatorname{Fix}(T) .
\end{aligned}
$$


Using (4.16), (4.17), and (4.20), we have

$$
\limsup _{n \rightarrow \infty}\left\langle p-S p, w_{n}-p\right\rangle \leq 0, \quad \forall p \in \operatorname{Fix}(T) .
$$

By using the fact that $\left\{w_{n}\right\}$ weakly converges to $x^{*}$, we have $\left\langle(I-S) p, x^{*}-p\right\rangle \leq 0, \forall p \in \operatorname{Fix}(T)$. As the same in (3.32), we find that

$$
\left\langle(I-S) x^{*}, x^{*}-p\right\rangle \leq 0, \quad \forall p \in \operatorname{Fix}(T) .
$$

That is, $x^{*} \in \Psi$. Thus $x^{*} \in \Upsilon$.

Step 4. Finally, as the Step 4 in Theorem 3.1, we can easily conclude that $x_{n} \rightarrow x^{*}$, where $x^{*}=P_{\Upsilon} x_{0}$. This completes the proof.

\section{NUMERICAL EXPERIMENTS}

In this section, we use some numerical experiments to illustrate the computational performance of our proposed Algorithm (3.1) and Algorithm (4.1) by comparing them with the Algorithm (1.7) in [10]. It should be pointed out that we use the FOM Solver [4] and the function fmincon in MATLAB Optimization Toolbox to effectively calculate the projections onto $C_{n} \cap Q_{n}$ and $C_{n+1}$. All the programs are performed in MATLAB2018a on a PC Desktop Intel(R) Core(TM) i5-8250U CPU @ 1.60GHz 1.800 GHz, RAM 8.00 GB.

Example 5.1. As an example, we seek common solutions for variational inequality problems and nonlinear optimization problems. Let $C$ be a nonempty convex and closed subset of a real Hilbert space $H$. We consider the variational inequality problem (in short, VIP):

$$
\text { find } x^{*} \in C \quad \text { such that }\left\langle f\left(x^{*}\right), x-x^{*}\right\rangle \geq 0, \quad \forall x \in C,
$$

where $f: H \rightarrow H$ is a single-valued mapping. Denote by $\operatorname{VI}(C, f)$ the solution of the variational inequality problem. Set $T:=P_{C}$ and $S:=I-\gamma f$, where $0<\gamma<2 / L$ ( $L$ is the Lipschitz constant of mapping $f$ ). We know that $\operatorname{Fix}\left(P_{C}(I-\gamma f)\right)=\mathrm{VI}(C, f)$. Therefore, variational inequality problem (5.1) is a special case of hierarchical fixed point problems HFPP (1.2). In VIP (5.1), one takes $f: R^{2} \rightarrow R^{2}$ as follows:

$$
f(x, y)=(2 x+2 y+\sin (x),-2 x+2 y+\sin (y)), \quad \forall x, y \in R .
$$

The feasible set $C$ is given by $C=\left\{x \in R^{2} \mid-10 e \leq x \leq 10 e\right\}$, where $e=(1,1)^{\top}$. It is not hard to check that $f$ is Lipschitz continuous with constant $L=\sqrt{26}$ and 1-strongly monotone [9]. Therefore, (5.1) has a unique solution $x^{*}=(0,0)^{\top}$.

On the other hand, let us consider the following nonlinear optimization problem

$$
\min H(x)=1+x_{1}^{2}-e^{-x_{2}^{2}}+\|x\|_{1},
$$

where $x=\left(x_{1}, x_{2}\right)^{\top} \in R_{+}^{2}$. We know that the optimal solution of $H(x)$ is $x^{*}=(0,0)^{\top}$. Set $F(x)=1+x_{1}^{2}-e^{-x_{2}^{2}}$ and $G(x)=\|x\|_{1}$. Take $A(x)=\nabla F(x)=\left(2 x_{1}, 2 x_{2} e^{-x_{2}^{2}}\right)^{\top}$ and $B(x)=\partial G(x)$. It is easy to check that $A$ is $\frac{1}{2}$-inverse strongly monotone on $R_{+}^{2}$, and $G$ is convex and lower semicontinuous but not differentiable. We have

$$
(I+\mu B)^{-1}(x)=\max \left(0,1-\frac{\mu}{|x|}\right) x .
$$

Obviously, $\Upsilon=\Phi \bigcap \Psi \bigcap \operatorname{Fix}(S)=(0,0)^{\top} \neq \emptyset$. Therefore, $x^{*}=(0,0)^{\top}$. In all algorithms, set $\psi_{n}=\frac{1}{n+1}, v_{n}=\frac{1}{(n+1)^{2}}, \mu=0.5$ and $\gamma=1 / \sqrt{26}$, take inertial parameter $\delta_{n}=0.4$ in the 
Algorithm (3.1) and the Algorithm (4.1). Denote by $E_{n}=\left\|x_{n}-x^{*}\right\|_{2}$ the error of iterative algorithms and $E_{n}<10^{-3}$ the common stopping criterion. Let $x_{-1}=x_{0}, z_{0}$ be randomly generated by the MATLAB function $k \times \operatorname{rand}(m, 1)$ (where Case I: $k=1$, Case II: $k=10$, Case III: $k=100$, and Case IV: $k=1000$ ). The numerical results are reported in Table 1 and Fig. 1.

TABLE 1. Compare the number of iterations for Example 5.1

\begin{tabular}{cccc}
\hline Cases & Algorithm (3.1) & Algorithm (4.1) & Algorithm (1.7) \\
\hline I & 26 & 11 & 109 \\
II & 61 & 56 & 421 \\
III & 139 & 115 & 2099 \\
IV & 272 & 201 & $>5000$ \\
\hline
\end{tabular}

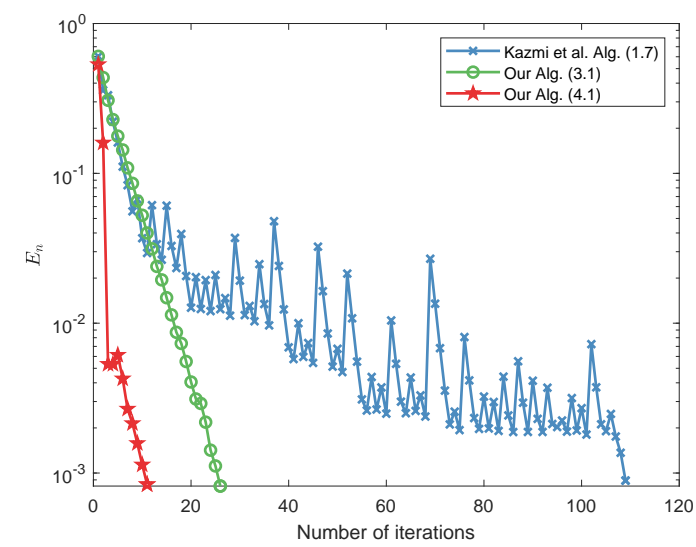

(a) Case I

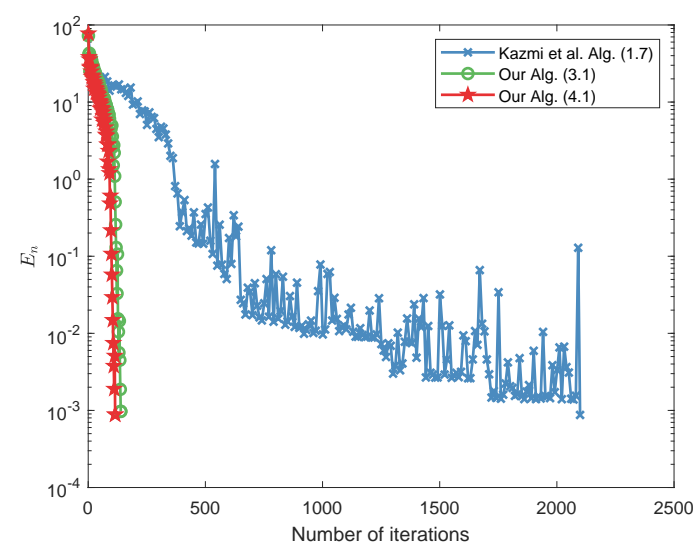

(c) Case III

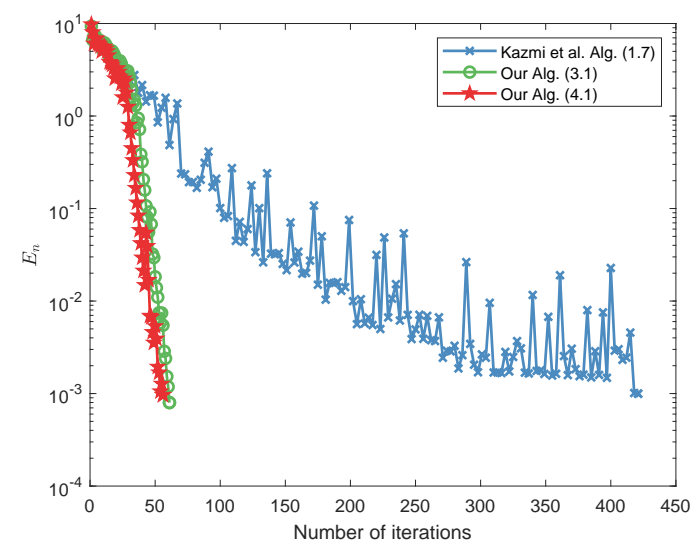

(b) Case II

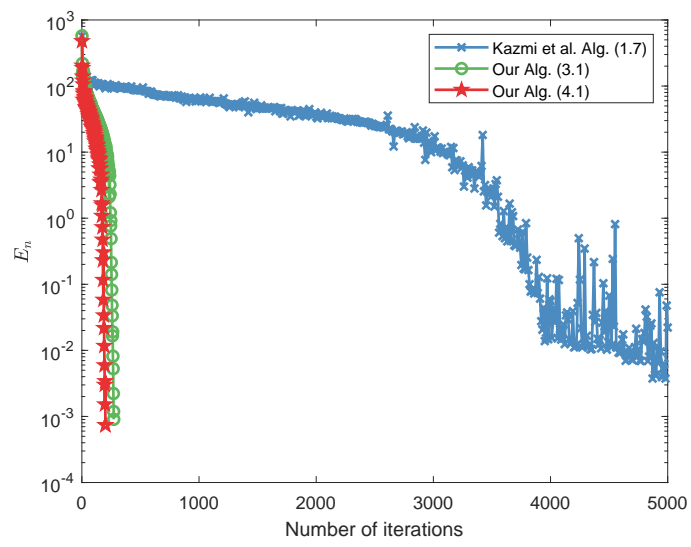

(d) Case IV

FIGURE 1. Convergence behavior of iteration error $\left\{E_{n}\right\}$ for Example 5.1

Example 5.2. In this example, we find common solutions for variational inequality problems and least squares optimization problems. First, in VIP (5.1), we consider the linear operator 
$f(x)=M x+q$, where $q \in R^{m}$ and

$$
M=N N^{\top}+U+D,
$$

where $N$ is a $m \times m$ matrix, $U$ is a $m \times m$ skew-symmetric matrix, and $D$ is a $m \times m$ diagonal matrix with its diagonal entries being nonnegative (hence $M$ is positive symmetric definite). The feasible set $C$ is given by $C=\left\{x \in R^{m}:-5 \leq x_{i} \leq 5, i=1, \ldots, m\right\}$. It is clear that $f$ is monotone and Lipschitz continuous with constant $L=\|M\|$. In this experiment, all entries of $N, U$ are generated randomly and uniformly in $[-5,5]$ and $D$ is generated randomly in $[1,5]$. Let $q=0$. Then, the solution set is $\{\mathbf{0}\}$.

On the other hand, let us consider the following least squares optimization problem via

$$
\min H(x)=\frac{1}{2}\|x-e\|_{2}^{2}+\|x\|_{1},
$$

where $x \in R^{m}, e=(1,1, \ldots, 1)^{\top} \in R^{m}$. We know the optimal solution of $H(x)$ is $x^{*}=(0,0, \ldots, 0)^{\top}$. Setting $F(x)=\frac{1}{2}\|x-e\|_{2}^{2}$ and $G(x)=\|x\|_{1}$. Taking $A(x)=\nabla F(x)=(x-e)$ and $B(x)=\partial G(x)$. It is easy to check that $A$ is 1 -inverse strongly monotone, and hence $\mu \subset(0,2)$. Taking $T=P_{C}$ and $S=I-\gamma f$. Obviously, we get that $\Upsilon=\Phi \bigcap \Psi \bigcap \operatorname{Fix}(\mathrm{S})=(0,0, \ldots, 0)^{\top} \neq \emptyset$ and hence $x^{*}=(0,0, \ldots, 0)^{\top}$. In all algorithms, set $\psi_{n}=\frac{1}{n+1}, v_{n}=\frac{1}{(n+1)^{2}}, \mu=1$ and $\gamma=1 / L$, take $\delta_{n}=0.4$ in the Algorithm (3.1) and the Algorithm (4.1). Let $x_{-1}=x_{0}, z_{0}$ be randomly generated by the MATLAB function $10 \times \operatorname{rand}(m, 1)$. Iterative error $E_{n}$ and stopping criterion are the same as in Example 5.1. We test the convergence behavior under different dimensional parameters $m$. The numerical results are reported in Table 2 and Fig. 2.

TABLE 2. Compare the number of iterations for Example 5.2

\begin{tabular}{cccc}
\hline Dimensional & Algorithm (3.1) & Algorithm (4.1) & Algorithm (1.7) \\
\hline 2 & 26 & 16 & 223 \\
5 & 105 & 66 & 540 \\
10 & 281 & 188 & 759 \\
20 & 877 & 734 & 1070 \\
\hline
\end{tabular}

Example 5.3. In this example, we consider common solutions for variational inequality problems and mixed equilibrium problems. First, in VIP (5.1), $f:=\left(a_{i j}\right)_{1 \leq i, j \leq m}$ is an $m \times m$ square matrix whose terms are given by

$$
f_{i j}= \begin{cases}1, & \text { if } j=m+1-i \text { and } j<i \\ -1, & \text { if } j=m+1-i \text { and } j>i \\ 0, & \text { otherwise }\end{cases}
$$

The feasible set is given by $C:=\left\{-5 \leq x_{i} \leq 5, i=1,2, \ldots, m\right\}$. It is clear that $f$ is Lipschitz continuous with constant $L=1$ and $x^{*}=(0,0, \ldots, 0)^{\top}$ is the solution. The mixed equilibrium problem (in short, MEP) is described as follows

$$
\text { find } x^{*} \in C \quad \text { such that } B\left(x^{*}, x\right)+\left\langle A x^{*}, x-x^{*}\right\rangle \geq 0, \quad \forall x \in C,
$$

where $B: C \times C \rightarrow R$ is a bifunction and $A: C \rightarrow H$ is a nonlinear mapping. Let $\Omega$ be the solution set of MEP (5.4). The MEP (5.4) is a special case of the MVIP (1.1) [10]. Let $A: R^{m} \rightarrow R^{m}$ be 


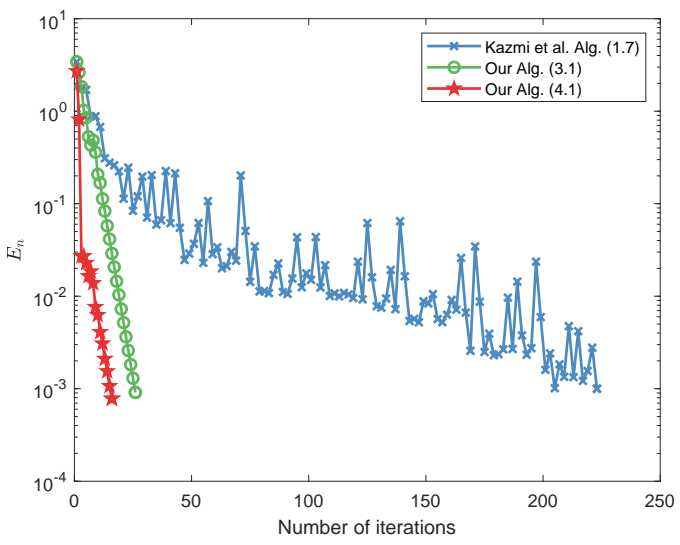

(a) Dimensional $m=2$

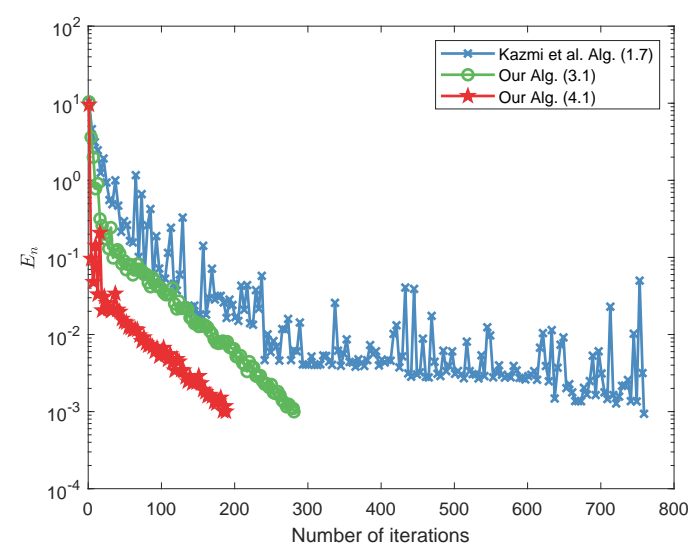

(c) Dimensional $m=10$

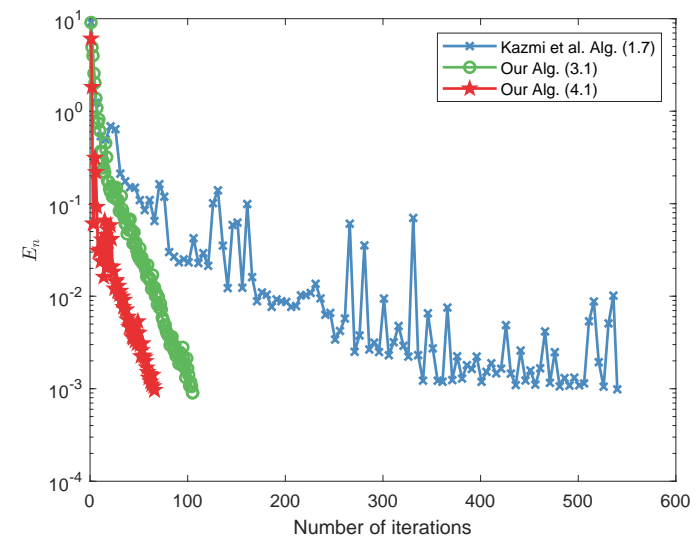

(b) Dimensional $m=5$

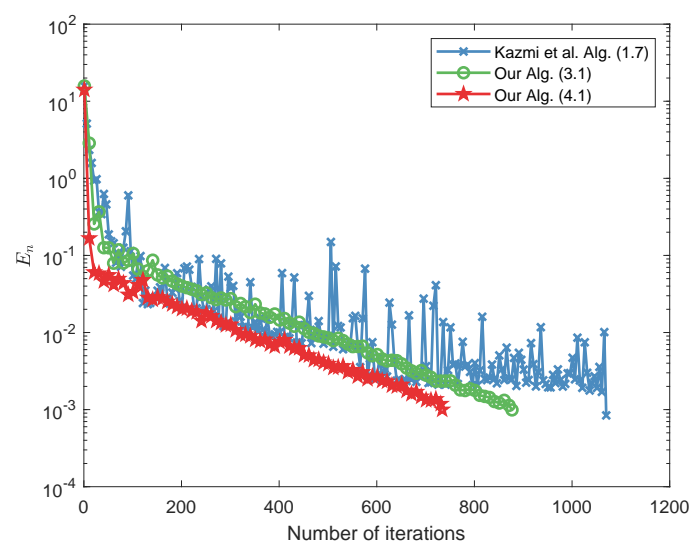

(d) Dimensional $m=20$

FIGURE 2. Convergence behavior of iteration error $\left\{E_{n}\right\}$ for Example 5.2

defined by $A=3 x$ and $B: R_{+}^{m} \times R_{+}^{m} \rightarrow R^{m}$ be defined by $B(x, y)=x(y-x)$. It is easy to get that $A$ is $\frac{1}{3}$-inverse strongly monotone, and hence $\mu \subset\left(0, \frac{2}{3}\right)$. Let $T=P_{C}$ and $S=I-\gamma f$. Obviously, one has $\Upsilon=\Omega \bigcap \Psi \bigcap \operatorname{Fix}(\mathrm{S})=(0,0, \ldots, 0)^{\top} \neq \emptyset$. Hence $x^{*}=(0,0, \ldots, 0)^{\top}$. In all algorithms, set $m=10, \psi_{n}=\frac{1}{n+1}, v_{n}=\frac{1}{(n+1)^{2}}, \mu=\frac{1}{4}$ and $\gamma=1$, take $\delta_{n}=0.4$ in the Algorithm (3.1) and the Algorithm (4.1). Let $x_{-1}=x_{0}, z_{0}$ be randomly generated by the MATLAB function $k \times \operatorname{rand}(m, 1)$ (where Case I: $k=1$, Case II: $k=10$, Case III: $k=50$, and Case IV: $k=100$ ). Iterative error $E_{n}$ and the stopping criterion are the same as in Example 5.1. We test the convergence behavior under different initial values. The numerical results are reported in Table 3 and Fig. 3.

Remark 5.1. (1) From Example 5.1-Example 5.3, we know that our proposed Algorithm (3.1) and Algorithm (4.1) are easy to implement, efficient and robust.

(2) From Table 1-Table 3 and Fig. 1-Fig. 3, we find that the inertial shrinking projection Algorithm (4.1) converges faster than the inertial shrinking projection algorithm without extrapolating step (3.1) and the hybrid iterative Algorithm (1.7). In addition, our proposed Algorithm (3.1) and Algorithm (4.1) are significantly faster than Algorithm (1.7), and the 
TWO INERTIAL PROJECTION ALGORITHMS

TABLE 3. Compare the number of iterations for Example 5.3

\begin{tabular}{cccc}
\hline Cases & Algorithm (3.1) & Algorithm (4.1) & Algorithm (1.7) \\
\hline I & 363 & 323 & 391 \\
II & 488 & 470 & 650 \\
III & 681 & 656 & 2081 \\
IV & 723 & 701 & 4109 \\
\hline
\end{tabular}

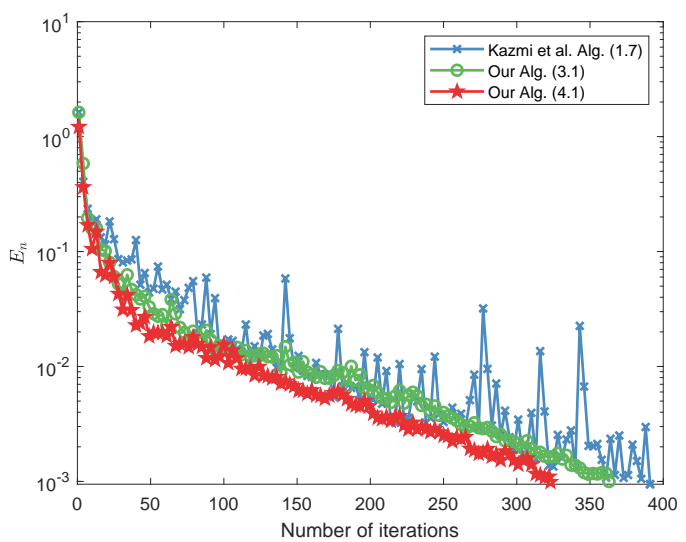

(a) Case I

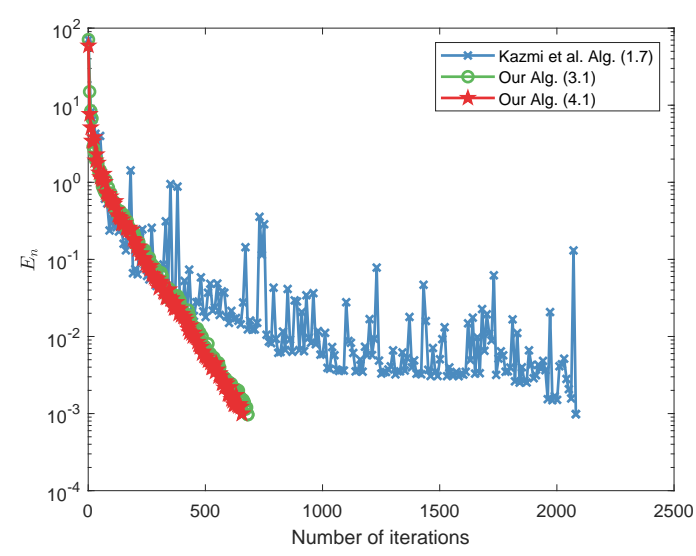

(c) Case III

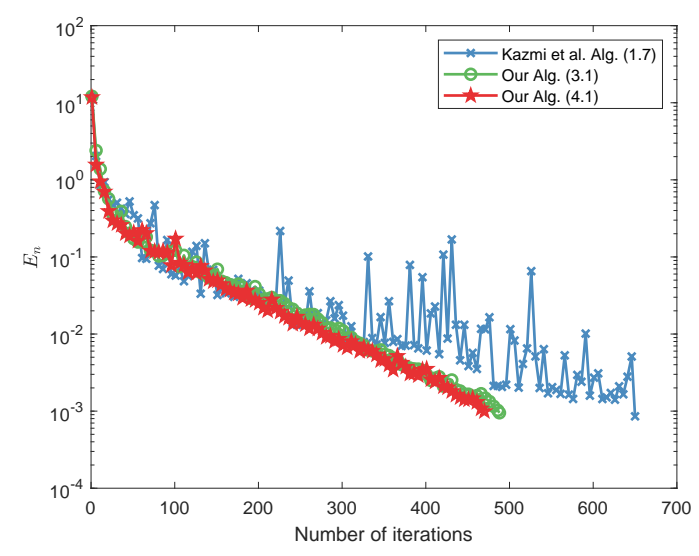

(b) Case II

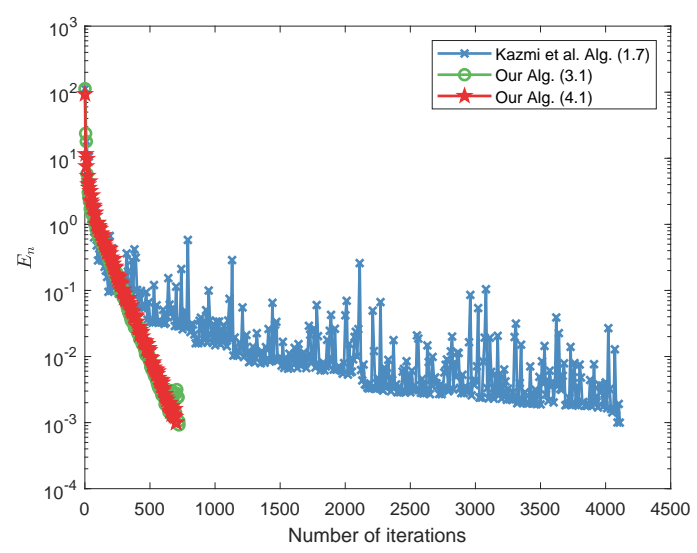

(d) Case IV

FIGURE 3. Convergence behavior of iteration error $\left\{E_{n}\right\}$ for Example 5.3

choice of initial values and the scale of the dimension do not affect the computational performance of our algorithms.

(3) Our shrinking projection iterative Algorithm (3.1) and Algorithm (4.1) have less oscillation and enjoy faster convergence speed than the hybrid iterative Algorithm (1.7). 


\section{REFERENCES}

[1] N.T. An, N.M. Nam, X. Qin, Solving $k$-center problems involving sets based on optimization techniques, J. Global Optim. 76 (2020), 189-209.

[2] H.H. Bauschke, P.L. Combettes, Convex Analysis and Monotone Mperator Theory in Hilbert Spaces, Springer, Berlin, 2011.

[3] A. Beck, M. Teboulle, A fast iterative shrinkage-thresholding algorithm for linear inverse problems, SIAM J. Imaging Sci. 2 (2009), 183-202.

[4] A. Beck, N. Guttman-Beck, FOM-a MATLAB toolbox of first-order methods for solving convex optimization problems, Optim. Methods Softw. 34 (2019), 172-193.

[5] S.Y. Cho, S.M. Kang, Approximation of common solutions of variational inequalities via strict pseudocontractions, Acta Math. Sci. 32 (2012), 1607-1618.

[6] P.L. Combettes, V.R. Wajs, Signal recovery by proximal forward-backward splitting, Multiscale Model. Simul. 4 (2005), 1168-1200.

[7] Q-L. Dong, K.R. Kazmi, R. Ali, X.H. Li, Inertial krasnosel'skiǐ-Mann type hybrid algorithms for solving hierarchical fixed point problems, J. Fixed Point Theory Appl. 21 (2019), 57.

[8] Q.-L. Dong, Y.Y. Lu, A new hybrid algorithm for a nonexpansive mapping, Fixed Point Theory Appl. 2015 (2015), 37.

[9] Q-L. Dong, Y.J. Cho, L.L. Zhong, T.M. Rassias, Inertial projection and contraction algorithms for variational inequalities, J. Global Optim. 70 (2018), 687-704.

[10] K.R. Kazmi, R. Ali, S. Yousuf, M. Shahzad, A hybrid iterative algorithm for solving monotone variational inclusion and hierarchical fixed point problems, Calcolo 56 (2019), 34.

[11] L. Liu, B. Tan, S.Y. Cho. On the resolution of variational inequality problems with a double-hierarchical structure. J. Nonlinear Convex Anal. 21 (2020), 377-386.

[12] D. Lorenz, T. Pock, An inertial forward-backward algorithm for monotone inclusions, J. Math. Imaging Vision 51 (2015), 311-325.

[13] P.E. Maingé, A. Moudafi, Strong convergence of an iterative method for hierarchical fixed-point problems, Pacific J. Optim. 3 (2007), 529-538.

[14] A. Moudafi, Krasnoselski-Mann iteration for hierarchical fixed-point problems, Inverse Probl. 23 (2007), 1635.

[15] A. Moudafi, P.E. Maingé, Towards viscosity approximations of hierarchical fixed-point problems, Fixed Point Theory Appl. 2006 (2006), 95453.

[16] C. Martinez-Yanes, H-K. Xu, Strong convergence of the CQ method for fixed point iteration processes, Nonlinear Anal. 64 (2006), 2400-2411.

[17] Y.V. Malitsky, V.V. Semenov, A hybrid method without extrapolation step for solving variational inequality problems, J. Global Optim. 61 (2015), 193-202.

[18] X. Qin, N.T. An, Smoothing algorithms for computing the projection onto a Minkowski sum of convex sets, Comput. Optim. Appl. 74 (2019), 821-850.

[19] X. Qin, J.C. Yao, A viscosity iterative method for a split feasibility problem, J. Nonlinear Convex Anal. 20 (2019), 1497-1506.

[20] H. Raguet, J. Fadili, G. Peyré, A generalized forward-backward splitting, SIAM J. Imaging Sci. 6 (2013), 1199-1226.

[21] D.R. Sahu, J.C. Yao, M. Verma, K.K. Shukla, Convergence rate analysis of proximal gradient methods with applications to composite minimization problems, Optimization, (2020), 10.1080/02331934.2019.1702040.

[22] B. Tan, Z. Zhou, S. Li. Strong convergence of modified inertial Mann algorithms for nonexpansive mappings. Mathematics 8 (2020), 462.

[23] B. Tan, S. Xu, S. Li, Inertial shrinking projection algorithms for solving hierarchical variational inequality problems, J. Nonlinear Convex Anal. 21 (2020), 871-884.

[24] T. Yuying, S. Plubtieng, Strong convergence theorems by hybrid and shrinking projection methods for sums of two monotone operators, J. Inequal. Appl. 2017 (2017), 72. 